\title{
Multiscale approaches to describe mechanical responses induced by particle removal in granular materials
}

\author{
Étude par approches multi-échelles de la réponse mécanique d'un milieu granulaire \\ induite par l'extraction de ses particules \\ Luc Scholtès $^{\mathrm{a}, \mathrm{b}}$, Pierre-Yves Hicher ${ }^{\mathrm{b}}$, Luc Sibille ${ }^{\mathrm{b}}$ \\ a CSIRO - Earth Science E Resource Engineering, QCAT, 1 Technology Court, Pullenvale, QLD, 4069, Australia \\ b GeM Laboratory, University of Nantes, ECN, CNRS, BP 92101, 44321 Nantes cedex 3, France
}

\begin{abstract}
A multi-scale approach is proposed as an attempt to assess internal erosion induced effects on the mechanical properties of a granular medium. When submitted to internal flow, some particles tend to be removed from the initial granular material, leading to drastic changes in the microstructure. The medium is changing during its lifetime and this cannot be ignored in attempts to model its mechanical behavior. In this first analysis, the degradation of a granular assembly is simulated through the progressive removal of its finest particles for both isotropic and anisotropic stress states. A discrete element model as well as an analytical micromechanical model are used to compare induced deformations and properties changes along the erosion process. The shear strength and flow properties appear to be strongly modified by this extraction phase: both models highlight a change from a dilatant to a contractant behavior with the degradation as well as strong dependency of the overall stability on the mobilized friction level. In particular, results show how failure is triggered when particle removal occurs for mobilized frictions greater than the one reached at the critical state.
\end{abstract}

Keywords:

Granular media

Micromechanics

Erosion

Failure

Une approche multi-échelle est proposée dans le but d'étudier les effets de l'érosion interne sur les propriétés mécaniques d'un milieu granulaire. Dans cette première analyse, la dégradation d'un assemblage granulaire est simulée par la suppression progressive de ses particules les plus fines pour différents états de contrainte, isotropes et anisotropes. Un modèle aux élements discrets ainsi qu'un modèle micromécanique analytique sont utilisés dans le but de comparer les déformations et les modifications induites sur le milieu. La resistance au cisaillement et les propriétés d'écoulement du matériau semblent être significativement modifiées par un tel processus d'extraction : les deux modèles sont unanimes pour mettre en évidence le passage d'un comportement dilatant à un comportement contractant du matériau avec sa dégradation, ainsi q'une dépendance critique de sa stabilité globale selon le niveau du frottement mobilisé auquel l'extraction de ses particules solides est réalisée. En particulier, les resultats montrent une tendance à l'effondrement lorsque l'extraction intervient pour des frottements mobilisés supérieurs à celui atteint à l'état critique.

Mots-clés :

Milieux granulaires

Micromécanique

Érosion

Rupture 


\section{Introduction}

Removing solid elements from a granular material can have a drastic influence on the mechanical behavior of the considered medium by modifying its mechanical constitutive properties. For instance, concerning the internal erosion process, the detachment and transport of some solid particles due to water internal flow may result in strong local evolutions of the microstructure with catastrophic consequences at the scale of civil engineering structures such as embankment dams.

For a complete description of internal erosion, one has to consider that solid elements can be extracted from the initial skeleton, whereas others may be relocated through the medium by water flow (see [1] for a complete synthesis). Filter rules as the one proposed by Kenney and Lau [2] for example, are now well accepted when the design of structures such as dams is of concerned. However, experimental data of internal erosion tests [1] have proven that zones exist where solid elements brought by water flow are not filtered during their transport, leading to a change of the initial grading due to seepage induced particle removal. A challenging point is therefore to describe and to understand the relation between the local degradation of the microstructure in removal zones, as well as the resulting modification of the constitutive mechanical properties of the medium.

Some coupled models have been proposed in order to relate the mechanical behavior of the soil with the erosion process $[3,4]$, but few attempts have been made to study the relation between the removal of soil particles and its consequences at the mesoscopic and macroscopic scales [5,6]. In this article, we present such an analysis through a multi-scale approach based upon numerical experiments performed with two fundamentally different micromechanical models. The first one is a three-dimensional numerical model based on the discrete element method (DEM) as introduced by Cundall and Strack [7]. The second one is a micromechanical constitutive relation recently developed by Chang and Hicher [8] that relates interparticle forces and displacements to macroscopic stresses and strains. In both cases, stress-strain states can be accessed through numerical and analytical homogenizations respectively.

For both approaches, analyses are based on the simulated mechanical behavior of a granular assembly subjected to axisymmetric triaxial loadings. Starting from different initial stress states, particles the most likely erodible are progressively removed from the assembly, leading to a rearrangement of the microstructure. At the macroscopic scale, resulting strains and variations of mechanical properties are followed. An attempt to highlight the relevant mechanisms induced by particle removals is finally suggested from a practical point of view.

\section{DEM approach}

Simulations are performed through the Yade-OpenDEM platform [9]. The 3D numerical sample is a granular assembly composed of 10,000 spheres with an initial grading as presented in Fig. 1. Six rigid frictionless boundary walls allow one to control the stress-strain state through the definition of their positions at each time step. The interparticle interaction is modelled by a linear elastic relationship between forces and interparticle displacements, associated with a slip Coulomb model.

Particles are considered to be rigid, but can overlap. This overlap accounts for the deformation induced by contact forces through the definition of normal and tangential stiffnesses $k_{n}$ and $k_{t}$ such as $f_{n}=k_{n} u_{n}$, and $\mathrm{d} f_{t}=-k_{t} \mathrm{~d} u_{t}$, with $u_{n}$ and $u_{t}$ the normal and tangential relative displacements respectively, and $f_{n}$ and $f_{t}$ the corresponding normal and tangential forces.

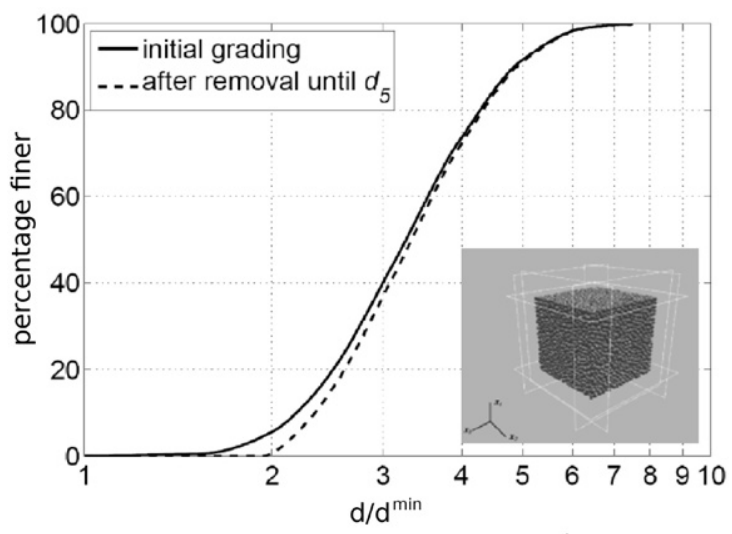

Fig. 1. Particle size distributions of the numerical sample before and after particle extractions. 
Table 1

Input parameters for the DEM.

\begin{tabular}{lll}
\hline $\begin{array}{l}\text { Normal stiffness } \\
k_{n} / D(\mathrm{MPa})\end{array}$ & $\begin{array}{l}\text { Stiffness ratio } \\
k_{t} / k_{n}\end{array}$ & $\begin{array}{l}\text { Friction angle } \\
\varphi\left({ }^{\circ}\right)\end{array}$ \\
\hline 250 & 0.5 & 35 \\
\hline
\end{tabular}
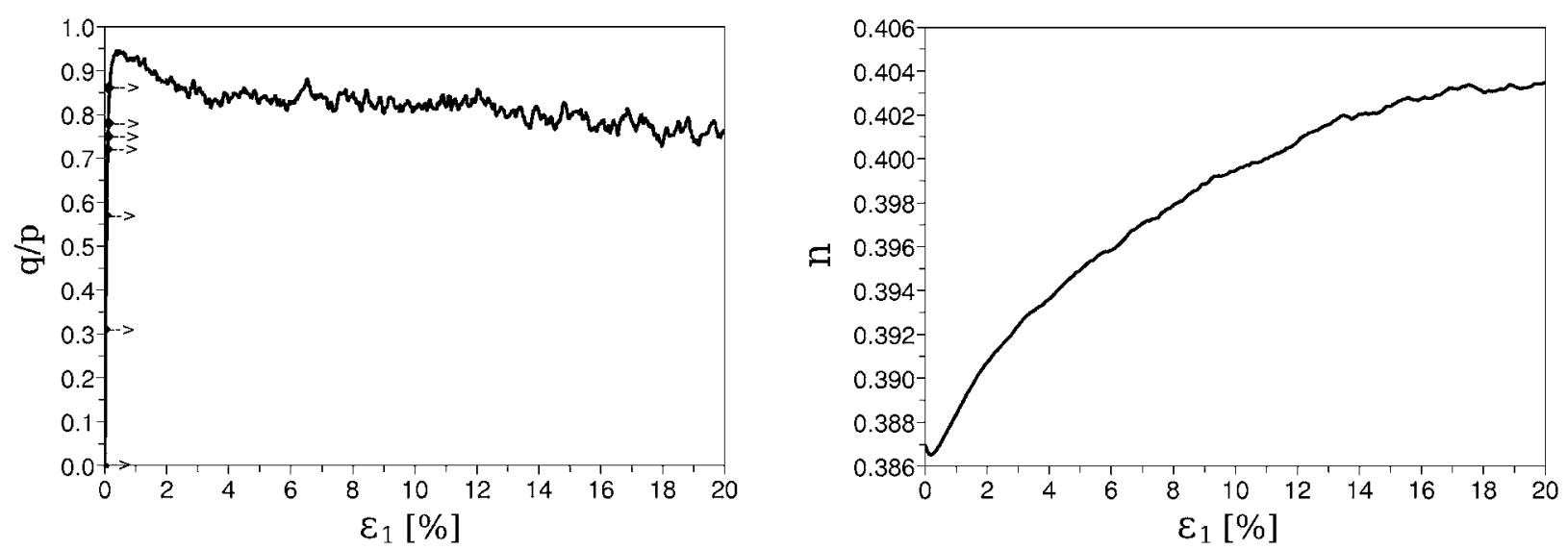

Fig. 2. Response of the intact numerical sample to triaxial compression loading for a $100 \mathrm{kPa}$ confining pressure. Arrows indicate stress states from which particle extractions are processed.

According to Coulomb friction, tangential forces are limited to a maximum value fixed by the interparticle friction angle $\varphi$ such as $\left\|f_{t}\right\| \leqslant f_{n} \tan \varphi$. Finally, the medium being considered as purely frictional, no tensile or cohesive forces are allowed at contacts. Table 1 gives the microscopic properties of the model used in this study. It is to be noted that stiffnesses are dependent functions of the particle size so as to avoid any scale effects. All the simulations are conducted without gravity in order to investigate the initial and changing fabric due only to changes in macroscopic stresses. The specimen is prepared through an isotropic-compaction technique which ensures the initial homogeneity of the packing. Particles are first randomly positioned inside the cubic box such as no overlaps occur between them. Particle radii are then increased progressively until the pressure generated on the boundary walls reaches the desired value. The final density of the packing can be controlled through the interparticle friction angle $\varphi_{c}$ chosen during this compaction phase. The smaller $\varphi_{c}$, the denser the resulting packing will be. Once the equilibrium of the assembly is verified, $\varphi_{c}$ is changed instantaneously to its testing value $\varphi=35^{\circ}$ which is kept constant for all the simulations. The packing is then allowed to stabilize under the applied isotropic stress of $100 \mathrm{kPa}$ before the prescribed loading to be applied. In this isotropic state, the granular assembly is characterized by a porosity $n=0.387$ and a coordination number $z=5.36$.

Fig. 2 provides the response of the assembly when subjected to an axisymmetric triaxial compression in terms of stresses and strains. The initial specimen behaves like a typical dense granular material with strain softening associated to dilatancy. As indicated in Fig. 2, the sample has been subjected to a series of particle extraction from different $\eta$ values representing the stress ratio between the differential stress $q$ and the mean pressure $p$ such as:

$$
\eta=\frac{q}{p}=3 \frac{\sigma_{1}-\sigma_{3}}{\left(\sigma_{1}+2 \sigma_{3}\right)}
$$

with $\sigma_{1}$ and $\sigma_{3}$ the major and minor principal stresses ( $\sigma_{2}=\sigma_{3}$ due to axisymmetric conditions).

\subsection{Extraction process}

As stated earlier, the aim of this study is not to provide an accurate estimation of internal erosion phenomena. Only a fully coupled hydro-mechanical model should be able to properly describe such a complex process with an accurate description of both detachment and transport of solid particles. However, as the focus here is on the potential role of particle removal on the macroscopic response of a granular assembly, a simplified approach has been developed in order to mimic particle removal due to internal erosion based upon two different assumptions.

First, it does not seem surprising that seepage flow picks preferentially on the less locked particles of the assembly. The less grains are subjected to interparticle forces, the more easily they can be detached from the granular skeleton. The less locked particles are thus the first potentially eligible for the transport process. In addition, particles should be small enough to be transported throughout the pore network. The smallest particles from the less locked (or loaded) ones are thus the most likely erodible. Interestingly, in purely frictional granular assemblies, the strongest contact forces are carried by the largest particles whereas the smallest particles are involved in the weakest contact forces [10]. 
From these observations, two different extraction processes have been defined:

- The first one is based on particle sizes only, and consists in removing the smallest particle contained in the assembly as suggested in $[5,6]$;

- The second one is based on both the size of the particles and their degree of interlocking, and consists in removing the less loaded of the smallest particles [11].

If sizes of particles are easily accessible in DEM simulations, one has to be defined how to determine their respective degree of loading. In the following, rather than computing a stress value at the particle scale, the degree of interlocking of a particle $p$ is estimated through the assessment of its mean internal moment $m^{p}$, defined as

$$
m^{p}=\operatorname{tr}\left(\boldsymbol{M}^{p}\right)
$$

where $\boldsymbol{M}^{p}$ is the internal moment tensor [12] given by

$$
M_{i j}^{p}=\sum_{\alpha \in p} r_{i}^{\alpha} f_{j}^{\alpha}
$$

with $\vec{r}$ the vector connecting the center of particle $p$ to the contact point associated with the contact force $\vec{f}$ involved in the contact $\alpha$.

According to Moreau [13], $\boldsymbol{M}^{p}$ gives a good evaluation of the stress at the grain scale as it is linked to the Cauchy stress $\sigma$ tensor by:

$$
\boldsymbol{\sigma}=\frac{1}{V} \sum_{p \in V} \boldsymbol{M}^{p}
$$

for a granular assembly with a volume $V$. Nevertheless, contrary to $\boldsymbol{\sigma}, \boldsymbol{M}^{p}$ has the particularity to be properly defined whatever the considered scale since the total internal moment $\boldsymbol{M}$ of a volume $V$ is simply given by:

$$
\boldsymbol{M}=\sum_{p \in V} \boldsymbol{M}^{p}
$$

$m^{p}$ is therefore assumed to give a good estimation of particles interlocking. In this study, the particle bearing the minimum $m^{p}$ value is thus considered as the most likely erodible of the assembly.

The two extraction procedures were both performed on the specimen at equilibrium for different values of the mobilized friction characterized by $\eta$ (Eq. (1)). In order to limit the extension of the process, the choice was made arbitrarily to allow the removal of up to $5 \%$ of the initial solid fraction, considering the smallest particles in the assembly. The eroded fraction $f e$ is defined as a function of the initial mass of the specimen such as

$$
f e=m_{\text {extracted }} / m_{\text {initial }}
$$

During extraction, wall positions are controlled in order to keep constant the prescribed stresses, and the process is driven such that equilibrium is reached before any subsequent particle is removed. Between two particle removals, the system evolves around quasi-static equilibrium with bursts of kinetic energy which intensity is linked to the role of the removed particle on the overall stability (characterized by $\boldsymbol{M}^{p}$ ). It is to be noted that kinetic energy variations and subsequent induced strains depend on the deviatoric stress level from which extraction is run. Fig. 3 presents the volumetric strain of the sample versus the percentage of extracted mass fe for both extraction criteria. In each case, the same qualitative response is observed. During the first stage of the extraction process, the sample first does not exhibit any significant volumetric changes whatever the $\eta$ value at initiation. Then, two distinct behaviors can be observed. First, for low $\eta$ values $(\eta<0.72)$, contractancy occurs with particle removal and the sample reaches a final equilibrium state after the entire extraction process ( $5 \%$ of solid mass removed). On the other hand, for $\eta \geqslant 0.72$, there is a transition from a contractant to a dilatant behavior which is associated with a loss of stability of the sample. Indeed, dilatancy occurs for a constant extracted mass value, indicating that no new equilibrium state is reached. Interestingly, the higher the mobilized friction $\eta$ is when extraction begins, the less extracted mass is needed to lead the sample to failure.

Comparing now both criteria one can note that, when particles are removed with respect to the unique size criterion, volumetric strains occur earlier during the extraction process. As shown in Figs. 4 and 3 for extractions performed in the isotropic state $(\eta=0)$, the macroscopic response of the specimen is directly related to the respective degree of interlocking of each removed particle. For instance, even if the selected particles belong to the smallest part of the grading, they can nevertheless participate to the stability of the whole assembly. As stated by [10], although bearing the weakest contact forces, the smallest particles can participate into the locking of the biggest ones involved in the main force chains.

Strikingly, when the loading of particles is taken into account in the extraction process, a certain amount of the solid mass $(f e \simeq 2.2 \%$ ) can be removed without any consequence at the macroscopic scale due to the presence of floating grains not involved in the force network $\left(m_{p}=0\right)$. By far, this feature is directly correlated to the absence of gravity in our simulations, but, even with gravity applied, those grains would not have any consequence on the global behavior of the specimen. 

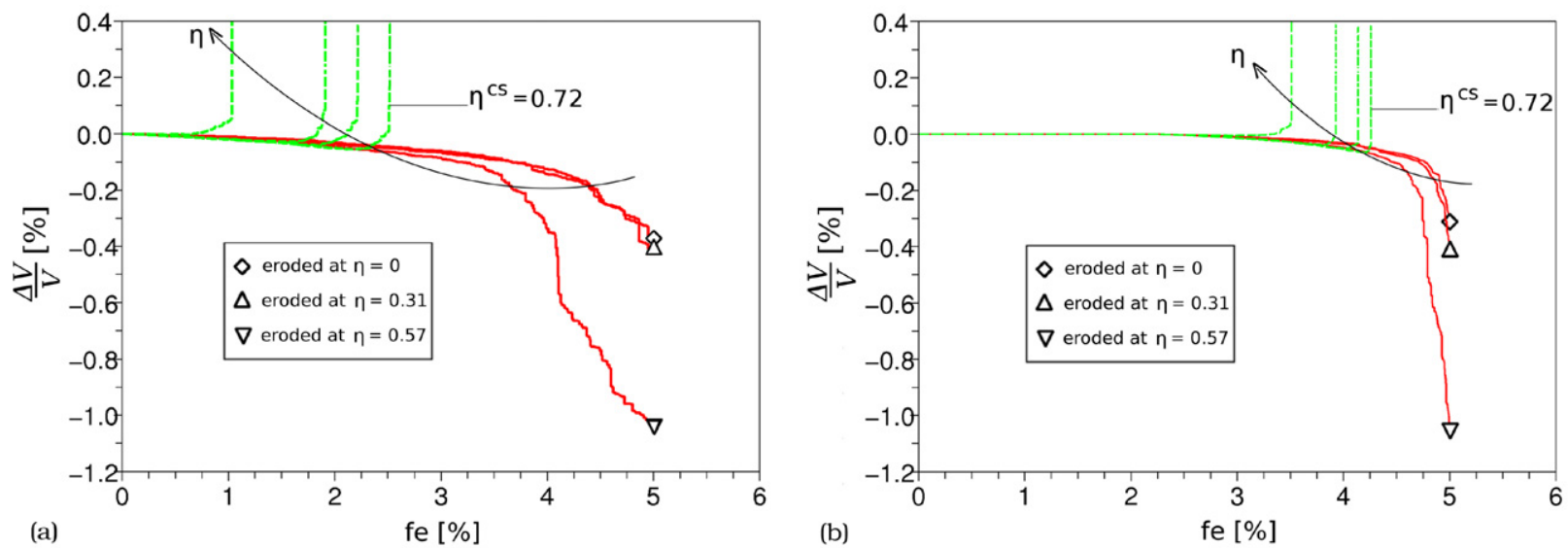

Fig. 3. Volumetric strains as a function of extracted mass $f e$ for particle removals performed at different mobilized frictions $\eta=q / p=\{0 ; 0.31$; $0.57 ; 0.72 ; 0.75 ; 0.78 ; 0.86\}$ : (a) size criterion only, (b) combined size and loading criteria. Solid lines indicate extraction processes leading to stable configurations after the entire $5 \%$ mass extraction (located with symbols), whereas dashed lines indicate extraction processes leading to sample failure.
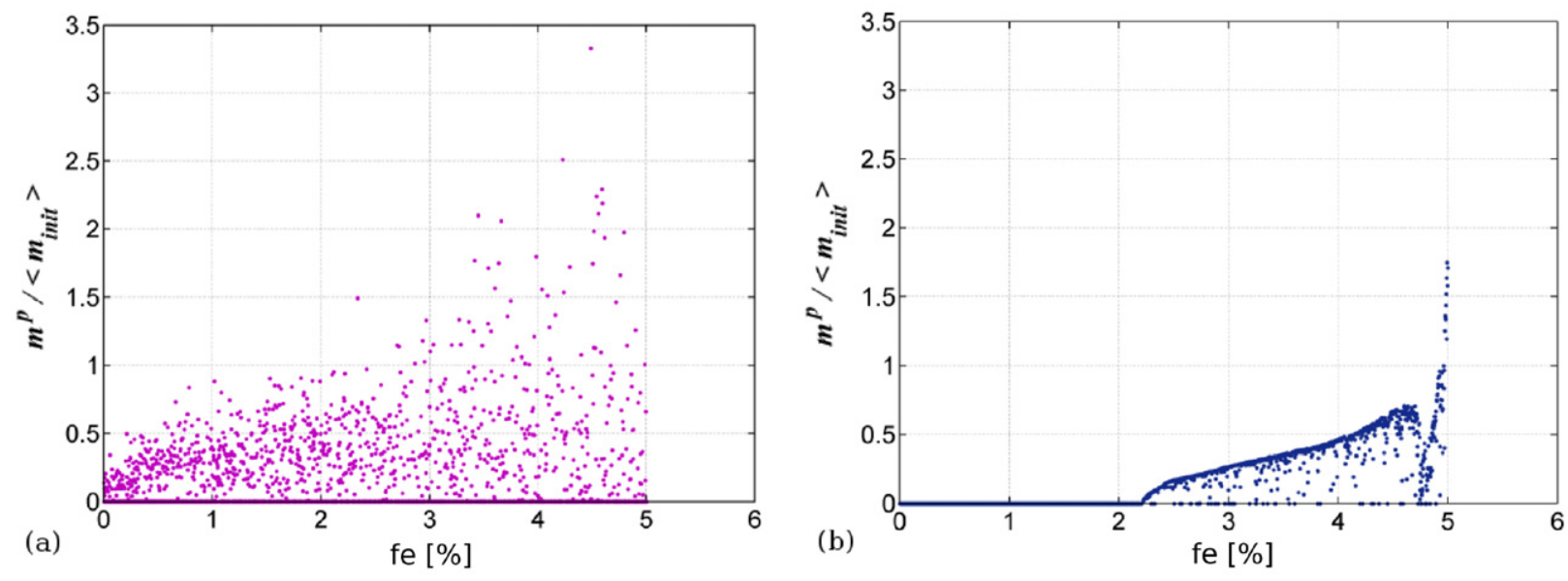

Fig. 4. Values of the normalized mean internal moment of each removed particles for particle extractions done in the isotropic state $(\eta=0)$ : (a) size criterion only, (b) combined size and loading criterion.

Obviously, this fe value is peculiar to the considered assembly and cannot therefore be treated as specific or representative. More interestingly, as presented in Fig. 3 as well as in Fig. 7, some fe values can be identified as the triggering factor for the development of large macroscopic strains. As stated earlier, the threshold value tends to diminish with the mobilized friction $\eta$, but no particular values can be readily emphasized. The process is however similar for every mechanical state: after the triggering of an important macroscopic event, important grain rearrangements occur in the microstructure, leading to a new stable or unstable mechanical state of the specimen depending on both the degree of degradation and the mobilized friction. For example, as presented in Fig. 4 for $\eta=0$, a sudden decrease of the minimum $m_{p}$ value can be observed for $f e \simeq 4.8 \%$. For this particular state, the sample suffers a contractancy which leads to a new stable configuration, but such rearrangements can also lead to dilatancy as for the higher mobilized frictions (see Fig. 3). Finally, in every case, material response results from the succession of locally induced grain reorganizations triggered by particle removal.

For both criteria, the simulated responses are qualitatively identical and the leading parameter seems to be more the set of particles potentially removed than the order of removal. However, contrary to the random effect involved with the unique size criterion, particle removal is hierarchically controlled with the combined size and loading criterion, in regards to flow potential wrenching properties. Consequently, in order to avoid any possible randomness in the following simulations, we will consider only the extraction process based on both the size and the loading of particles.

\subsection{Material response}

As shown in Fig. 3, depending on the mobilized friction from which extractions are conducted, the sample tends to compress and stabilize or to dilate and fail. It is remarkable that the limiting threshold for the sample to fail corresponds to the residual state $\left(\eta^{c s}=0.72\right)$ obtained for large shear deformations of the intact specimen - the so-called critical state [14] - as shown in Fig. 5. Indeed, for shear stress state inferior to the critical state $\left(\eta<\eta^{c s}=0.72\right)$, particle removal is 

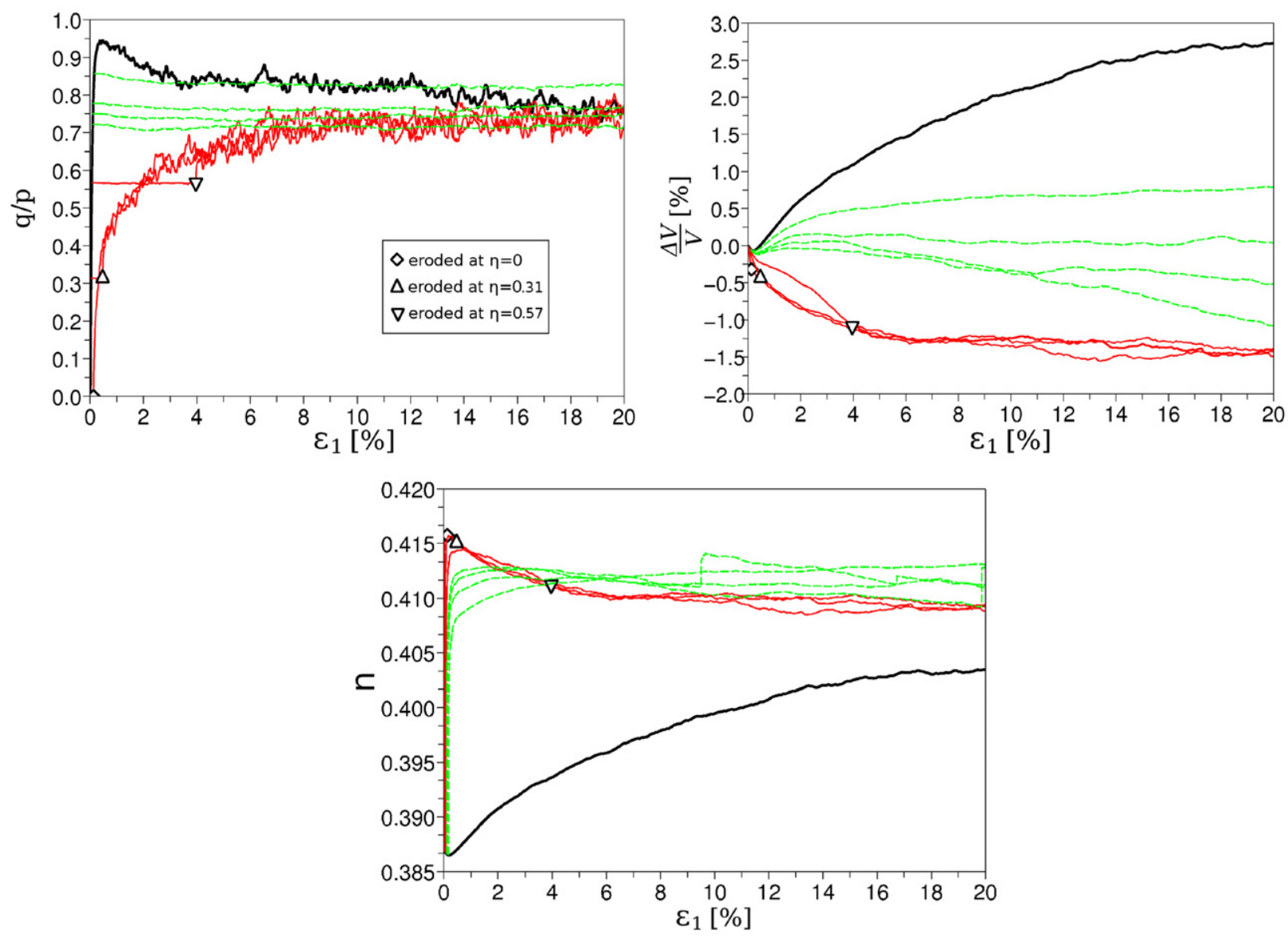

Fig. 5. DEM simulations of particle extraction for different mobilized friction $\eta=q / p=\{0 ; 0.31 ; 0.57 ; 0.72 ; 0.75 ; 0.78 ; 0.86\}$ and following triaxial compressions realized on an initially dense granular specimen (thick black line). Solid thin lines indicate extraction processes leading to stable configurations after the entire 5\% mass extraction (located with symbols), whereas dashed thin lines indicate extraction processes leading to sample failure.

associated with contractancy and the sample is able to reach a stable configuration after the 5\% extraction. On the contrary, for $\eta \geqslant \eta^{c s}=0.72$, particle removal induces a dilatancy of the sample which is associated with a loss of stability ( $\left.\varepsilon_{1}>20 \%\right)$.

Interestingly, one can see that the extraction process tends to increase the sample porosity whatever the induced dilatant or, more surprisingly, contractant behavior of the sample. Due to the marginal role of removed particles on the overall stability of the assembly, the initial microstructure supports an increase of its porosity with limited rearrangements. This result tends to confirm what Muir Wood et al. obtained in 2D [5]: the creation of a more open microstructure due to particle removals dominates the effect of sample compression induced by local destabilizations. The evolution of the porosity appears, therefore, as a pertinent parameter to characterize materials subjected to erosion phenomena, with a measurable overview of induced internal structural changes.

In order to bring all the specimens to failure, triaxial compressions have been continued for the three samples which stabilized after the entire extraction process (see Fig. 5). Intuitively, the increase of the initial porosity produces a change in the specimen behavior from a dilatant behavior to a contractant behavior, confirming therefore the pertinence of monitoring the porosity when considering mechanical properties of a potentially erodible material. A remarkable issue concerns the change of the porosity value for the critical state which is different from the degraded specimens compared to the intact one. According to what Muir Wood and co-authors [5,6] have observed, this change in the critical state properties is due to the change of grading induced by particle removal (Fig. 1).

When considering now the Mohr-Coulomb limit conditions obtained for both intact and degraded specimens (resulting from particle extractions conducted at three different isotropic states under 50, 100 and $200 \mathrm{kPa}$ respectively), it is obvious that the shear strength of the material is greatly affected by the removal of some of its constitutive particles (see Fig. 6). Particle extraction significantly weakens the medium by decreasing the internal friction angle from $24.2^{\circ}$ for the intact material to $20.8^{\circ}$ for the degraded one, corresponding, in this case, to its residual value (see Fig. 5). As shown in Figs. 5 and 6 , all these modifications in the characteristics of the mechanical response (internal friction angle, volumetric strain, residual state) are independent of the initial stress state at which particle extractions are conducted. Consequently, this tendency would mean that the modification of the mechanical properties to consider in order to predict the global behavior 


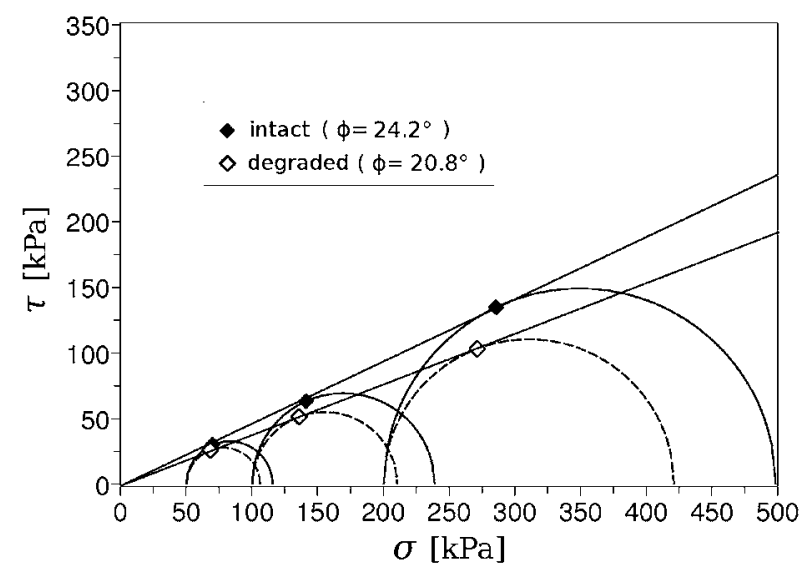

Fig. 6. Mohr-Coulomb failure envelopes corresponding to the DEM numerical sample before and after particle extraction.

of a structure does not depend on the mobilized strength in locations where erosion occurs, but only on the erosion progress status.

Despite the relevance of discrete element methods to give insights into constitutive properties of soils, the computational cost is still a limit, and it comes to some issues when one has to deal with real soil structures such as embankment dams. Thus, it is necessary to study the capability of approaches more able to deal with engineering boundary value problems such as analytical models for example. The following section aims at presenting the application of a constitutive micromechanical relationship to describe the mechanical responses pointed out here with the DEM.

\section{Analytical approach}

In this section, we first present the constitutive relation used to compare with the DEM model in terms of induced effects of particle extraction on the mechanical behavior. The approach is based on a homogenization technique applied to derive the stress-strain relationship of a granular assembly from forces and displacements at the particle level [8]. Key features of the particle removal modelling are then introduced before discussing simulation results in regards to the ones previously obtained with the DEM approach.

\subsection{Stress-strain model}

The basic idea is to view the packing as represented by a set of microsystems which correspond to the contact planes. A local constitutive law is introduced by considering a relationship between interparticle forces and displacements along a set of contact planes. The overall stress-strain relationship of the packing is then obtained from averaging the contact plane behavior.

The local constitutive law is assumed to be elastoplastic (see [8])

$$
\dot{f}_{i}^{\alpha}=k_{i j}^{\alpha p} \dot{\delta}_{j}^{\alpha}
$$

where $\alpha$ denotes the orientation of the given local plane.

The elastic part is derived from the Hertz-Mindlin's formulation. The yield function is assumed to be of the MohrCoulomb type, defined in a contact-force space by

$$
F\left(f_{i}, \kappa\right)=T-f_{n} \kappa\left(\Delta^{p}\right)=0
$$

where $\kappa\left(\Delta^{p}\right)$ is a hardening/softening parameter. The shear force $T$ and the rate of plastic sliding $\Delta^{p}$ are defined as

$$
T=\sqrt{f_{s}^{2}+f_{t}^{2}} \text { and } \Delta^{p}=\sqrt{\left(\delta_{s}^{p}\right)^{2}+\left(\delta_{t}^{p}\right)^{2}}
$$

The hardening function is defined by a hyperbolic curve in the $\kappa-\Delta^{p}$ plane, which involves two material constants $\Phi_{p}$ and $k_{p 0}$ such as

$$
\kappa=\frac{k_{p 0} \tan \Phi_{p} \Delta^{p}}{\left|f_{n}\right| \tan \Phi_{p}+k_{p 0} \Delta^{p}} \sigma_{i j}
$$

Plastic sliding often occurs along the tangential direction of the contact plane with an upward or downward movement; thus, shear-induced dilation/contraction takes place. Dilatancy effect can be described by

$$
\frac{\mathrm{d} \delta_{n}^{p}}{\mathrm{~d} \Delta^{p}}=\frac{T}{f_{n}}-\tan \Phi_{0}
$$


Table 2

Input parameters for the analytical model.

\begin{tabular}{llllll}
\hline$e_{\text {ref }}$ & $p_{\text {ref }}(\mathrm{MPa})$ & $\lambda$ & $\Phi_{\text {mu }}\left({ }^{\circ}\right)$ & $\Phi_{0}\left({ }^{\circ}\right)$ & \\
\hline 0.78 & 0.01 & 0.05 & 19 & 19 & 3 \\
\hline
\end{tabular}

where the material constant $\Phi_{0}$ can be considered in most case to be equal to the interparticle friction angle $\Phi_{\mu}$. On the yield surface, under a loading condition, the shear plastic flow is determined by a normality rule applied to the yield function. However, the plastic flow in the direction normal to the contact plane is governed by the stress-dilatancy equation in Eq. (11). Thus, the flow rule is non-associated.

Resistance against sliding on a contact plane depends on the degree of interlocking by neighboring particles. The resistance can be related to the packing void ratio $e$ by

$$
\tan \Phi_{p}=\left(\frac{e_{c}}{e}\right)^{m} \tan \Phi_{\mu}
$$

where $m$ is a material constant and $e_{c}$ corresponds to the critical void ratio for a given state of stress. For dense packing, $e_{c} / e$ is greater than 1 and, therefore, the apparent interparticle friction angle $\Phi_{p}$ is greater than the internal friction angle $\Phi_{\mu}$. When the packing structure dilates, the degree of interlocking and the apparent friction angle is reduced, which results in a strain-softening phenomenon. For loose packing, the apparent friction angle $\Phi_{p}$ is smaller than the internal friction angle $\Phi_{\mu}$ and increases during the material contraction.

The critical void ratio $e_{c}$ is a function of the mean stress applied to the overall assembly and can be written as follows:

$$
e_{c}=\Gamma-\lambda \log \left(p^{\prime}\right) \quad \text { or } \quad e_{c}=e_{\text {ref }}-\lambda \log \left(\frac{p^{\prime}}{p_{\text {ref }}}\right)
$$

where $\Gamma$ and $\lambda$ are two material constants, $p^{\prime}$ is the mean effective stress of the packing, and $\left(e_{r e f}, p_{\text {ref }}\right)$ is a reference point on the critical state line.

\subsection{Model parameters}

One can summarize the material parameters as:

- Normalized contact number per unit volume: $N l^{3} / V$.

- Mean particle size: $2 R$.

- Inter-particle elastic constants: $k_{n 0}, k_{t 0}$ and $n$.

- Inter-particle friction angle: $\Phi_{m u}$ and $m$.

- Inter-particle hardening rule: $k_{p 0}$ and $\Phi_{0}$.

- Critical state for packing: $\lambda$ and $\Gamma$ or $e_{\text {ref }}$ and $p_{\text {ref }}$.

Except for critical state parameters, all the parameters are related to interparticle contacts. Standard values for $k_{p 0}$ and $\Phi_{0}$ are the following: $k_{p 0}=k_{n 0}$ and $\Phi_{0}=\Phi_{m u}$ [8]. Therefore, for dry or saturated samples, only five parameters have to be obtained from experimental results and these can all be determined from the stress-strain curves obtained from triaxial tests.

The number of contacts per unit volume $\mathrm{Nl}^{3} / \mathrm{V}$ changes during the deformation. Using the experimental data by Oda [15] for three mixtures of spheres, we can approximate the total number of contacts per unit volume related to the void ratio by the following expression:

$$
\frac{N}{V}=\left(\frac{N}{V}\right)_{0} \frac{\left(1+e_{0}\right) e_{0}}{(1+e) e}
$$

where $e_{0}$ is the initial void ratio of the granular assembly. This equation is used to describe the evolution of the contact number per unit volume. The initial contact number per unit volume can be obtained by matching the predicted and experimentally measured elastic modulus for specimens with different void ratios $[8,16]$.

In this study, the normal stiffness and the ratio between tangential and normal stiffnesses are taken equal to the values considered in the DEM approach. The parameters of the plastic part of the contact law are given in Table 2. Parameters determining the critical state line of the DEM simulations are, as well as the value of $m$, calibrated by curve fitting using the test results shown in Fig. 2.

The numerical simulation of a monotonic triaxial test on an intact sample is plotted with a thick line in Fig. 8. One can see that the microstructural model is capable of reproducing by good accuracy the macroscopic stress-strain curve as well as the volume change obtained by DEM simulation. 


\subsection{Simulations of particle extraction}

What could be the impact of removing part of the solid fraction on the behavior of granular materials? Considering the homogenization technique presented in the previous section, two major influences can be noted. First, the number of interparticle contacts $N$ can be reduced, leading to a decrease of the normalized number of contacts per unit volume $\mathrm{Nl}^{3} / \mathrm{V}$. This decrease in the number of contacts will concentrate the contact forces on the remaining contacts and, therefore, create more interparticle displacements. By integrating these micro-displacements all over the elementary volume, we will obtain additional deformation of the granular assembly. The second consequence is linked to what we previously called the interlocking influence. The sliding resistance at each contact has two origins: one corresponds to the friction between two particles defined by the internal friction angle $\Phi_{\mu}$; the other is linked to a geometrical effect of the neighboring particles, which will resist against the sliding displacement of two particles in contact and all the more so if the packing is dense. Therefore, the pulling off of a fraction of the particles will release the interlocking influence and facilitate the contacting particles to slide together. In the model, this interlocking influence is taken into account by Eqs. (12) and (13), which relate the sliding resistance, controlled by the apparent friction angle $\Phi_{p}$, to the void ratio of the assembly. So, when part of the solid fraction is removed from the material, the void ratio increases and, as a consequence, the sliding resistance decreases, which can create additional interparticle displacements, and a greater deformation of the granular assembly.

The model cannot take into account the size of individual particles. Only the mean size value is considered as a model parameter. It is, therefore, not possible to model exactly the same process as the one used in the DEM simulations, which corresponds to the removal of the finest fraction. In this section, we will simply consider the removal of a given fraction fe of the solid particles as defined in Eq. (6). If one can assume that the particle density is the same for any particle size, Eq. (6) can be written in terms of volume:

$$
f e=V f / V s_{0}
$$

where $V f$ is the extracted solid volume and $V s_{0}$ is the initial solid volume per unit volume of soil. One can then relate $f e$ to the void ratio change due to the removed solid fraction

$$
f e=1-\frac{V s}{V s_{0}}
$$

where $V s$ is the solid volume after extraction.

The void ratio $e$ is given by

$$
e=\frac{(V-V s)}{V s}
$$

When no deformation takes place during the extraction of solid particles, the total volume remains constant and the volume occupied by the extracted particles is replaced by the same volume of voids. Therefore, we can write:

$$
\Delta e=e-e_{0}=V\left(\frac{1}{V s}-\frac{1}{V s_{0}}\right)
$$

Combining Eqs. (16) and (18), we obtain

$$
(\Delta e)_{e x}=\frac{f e\left(1+e_{0}\right)}{1-f e}
$$

where $e_{0}$ is the initial soil void ratio before the erosion process begins and $(\Delta e)_{e x}$ is the void ratio due to the extraction process.

When the extracted fraction fe increases progressively, it creates a change in the void ratio. This change is taken into account in the model by using Eqs. (11) and (12). If the material is subjected to a constant state of external stresses, the evolution of the sliding resistance introduced by Eq. (12) creates disequilibrium at each contact point leading to local sliding. All the local displacements are then integrated to produce the macroscopic deformation of the soil specimen. This macroscopic deformation induces a volumetric change $\varepsilon_{V}$ and therefore a change in the void ratio which is added to the void ratio change caused by particle removal (Eq. (19)).

$$
(\Delta e)=(\Delta e)_{e x}+\varepsilon_{V}(1+e)
$$

Therefore, the void ratio is calculated at the end of each step which consists, at first, to impose a change in void ratio corresponding to an incremental increase of the eroded fraction $\mathrm{fe}$, and then, to calculate the induced deformations. Under these circumstances, $f e$ can be considered as a parameter of the yield surface and the yield function (Eq. (8)) for each local plane becomes

$$
F\left(f_{i}, \kappa, f e\right)=T-f_{n} \kappa\left(\Delta^{p}, f e\right)=0
$$

The change of void ratio at the end of each step can be higher or lower than the one due to the sole removed solid fraction, depending on the sign of the volume change due to the induced deformations, as it will be shown later on. A contraction during this process will contribute to delay its impact, whereas a dilation will accelerate the deformation progress. 

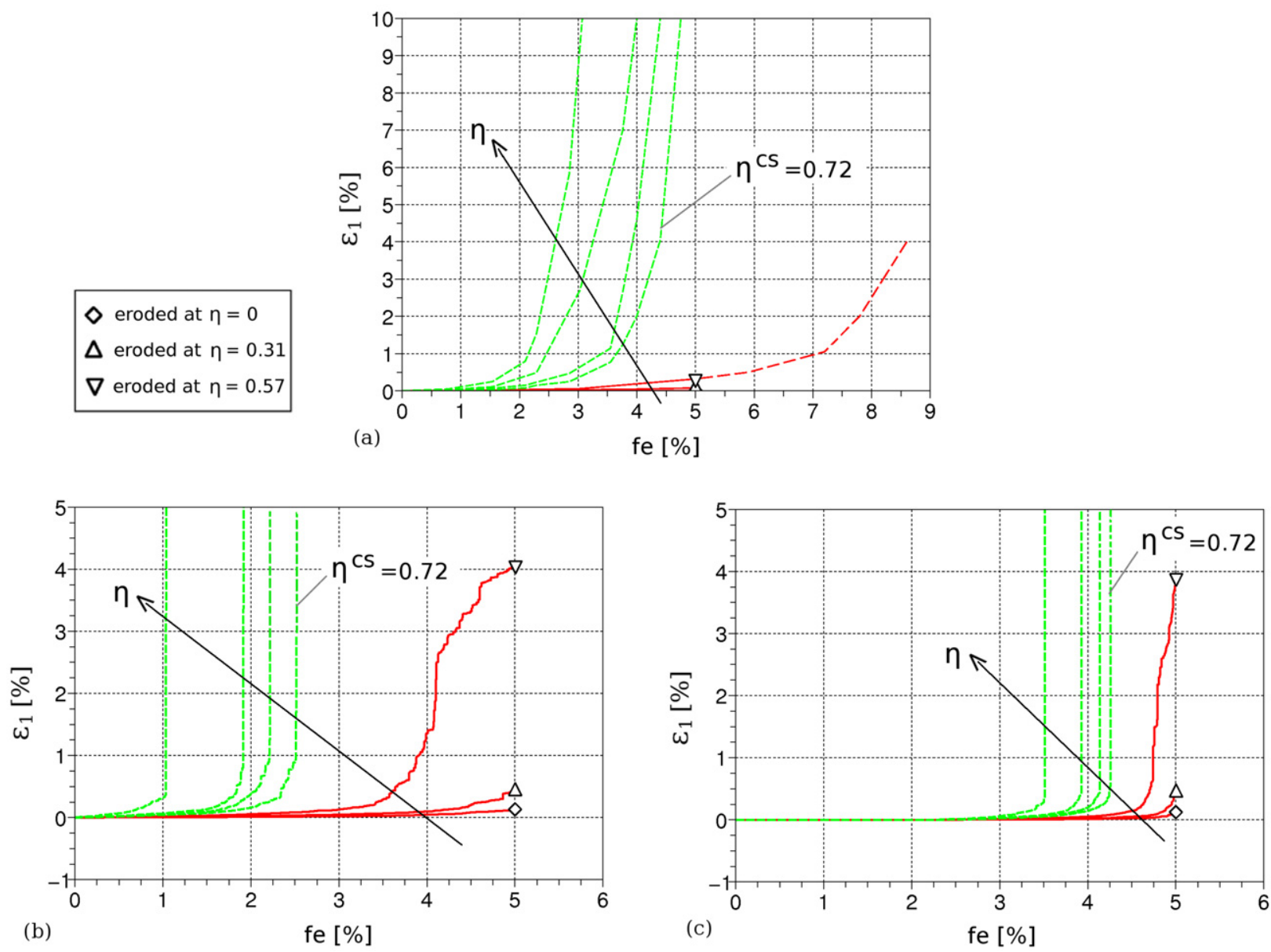

Fig. 7. Axial strain as a function of the extracted mass $f e$ for particle removals performed at different mobilized frictions $\eta=q / p=\{0 ; 0.31 ; 0.57 ; 0.72$; $0.75 ; 0.78 ; 0.86\}$ : (a) analytical model, (b) DEM with size criterion, (c) DEM with combined size and loading criterion. Solid thin lines indicate extraction processes leading to stable configurations after the entire 5\% mass extraction (located with symbols), whereas dashed thin lines indicate extraction processes leading to sample failure.

\section{Comparisons between analytical and DEM approaches}

Numerical simulations of particle extraction with the analytical model were performed from shear stress levels identical to those used with the DEM (see Fig. 2). In Fig. 7(a) is presented the evolution of the axial strain $\varepsilon_{1}$ as a function of the extracted mass ratio fe when particle extraction is carried out with the analytical model.

One can see that progressive deformations develop within the specimens during particle extraction. For high stress ratios $\eta$, the axial strain increases rapidly and leads to unlimited strains under a constant stress state. Actually, such a response of the model has made quite difficult the convergence of the numerical algorithm for axial strain values higher than $10 \%$. Consequently, in these cases, simulations were voluntarily stopped at $\varepsilon_{1}=10 \%$, and maximum strain values displayed in Fig. 7(a) constitute only lower bounds. Features described here above show that failure is obtained for these high stress ratios. On the contrary, for lower stress ratios, typically lower that the stress level reached at the critical state $\eta^{c s}=0.72$, the strain evolution is much slower and a large amount of solid mass is required to be extracted in order to develop noticeable strains.

The corresponding DEM results are plotted in Figs. 7 (b) and 7 (c) for the size criterion and the combined size and loading criterion, respectively. It is worth noting that, with the analytical model, failure occurs for intermediate $f e$ values (in the range between 2 and $4 \%$ according to the mobilized friction) situated between the lower bounds given by the DEM with the size criterion only ( $f e \approx 1$ to $2.5 \%$ ) and the upper bounds obtained with the combined size and loading criterion ( $f e \approx 3.5$ to $4.2 \%$ ). Hence, although separate particle size is not considered in the extraction process with the analytical model (actually the extraction process is conducted through the progressive decrease of the solid volume), the removed solid fraction fe needed to trigger failure with the analytical model belongs to the range given by DEM simulations. Interestingly, this last point could constitute a practical simplification for stability analysis of structures during erosion phenomena, avoiding to deal with the difficult issue concerning the time evolution of the grading of the soil. 

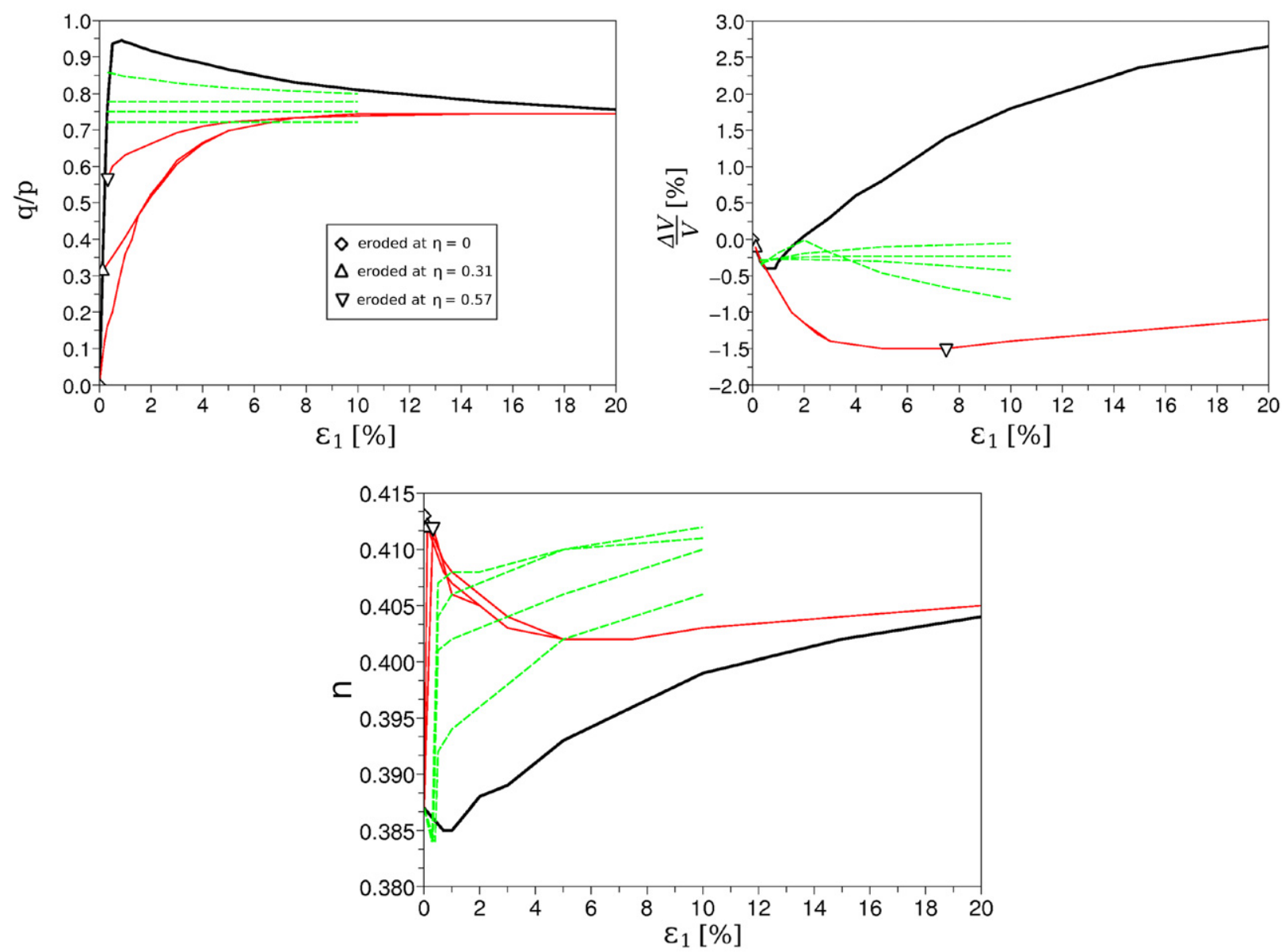

Fig. 8. Analytical simulations of particle extraction for different mobilized friction $\eta=q / p=\{0 ; 0.31 ; 0.57 ; 0.72 ; 0.75 ; 0.78 ; 0.86\}$ and following triaxial compressions realized on an initially dense granular specimen (thick black line). Solid thin lines indicate extraction processes leading to stable configurations after the entire $5 \%$ mass extraction (located with symbols), whereas dashed thin lines indicate extraction processes leading to sample failure.

As for the DEM, particle extractions modelled with the analytical model were stopped when fe $=5 \%$. Comparison of Figs. 7(a), 7(b) and 7(c) shows, when failure was not triggered (i.e. for $\eta=0,0.31$ and 0.57 ), that final resulting axial strains simulated with the analytical model are much lower than the ones computed with the DEM, with a difference of about one decade. For $\eta=0.57$ the extraction has been carried on until the axial strain became close to the one obtained by DEM simulations, i.e. $\varepsilon_{1} \approx 4 \%$ (see the dashed line in Fig. $7(\mathrm{a})$ ). To produce a similar strain with the analytical model, an eroded fraction $f e$ about 1.7 times larger than the limit of $5 \%$ fixed for the DEM is necessary. This difference may be related to a contractant behavior more developed for the analytical model than for the DEM (triaxial compressions on the intact specimen show a minimum volumetric strain of about $-0.4 \%$ for the analytical model (Fig. 8) whereas it is less than $-0.1 \%$ for the DEM (Fig. 2)). A more important contractancy leads to delaying the opening of the microstructure induced by solid extraction, limiting consequently shear strength reduction as well as straining. In cases where failure occurred during solid extraction, there is not such a difference since the aptitude involved in the specimen responses is their aptitude to dilate, and no more their aptitude to contract. In the same way as in DEM simulations, when the extraction process has not triggered failure, a subsequent monotonic compression was performed on the degraded specimen. A synthesis of specimen's responses during extraction processes and subsequent triaxial compressions is presented in Fig. 8 in $\left(q / p, \varepsilon_{1}\right),\left(\mathrm{d} V / V, \varepsilon_{1}\right)$ and $\left(n, \varepsilon_{1}\right)$ diagrams.

During extractions, the response strain path is linked to the initial mobilized friction. At high shear stress ratio, volume increases during extraction, whereas at smaller shear stress it decreases. This behavior is in agreement with DEM simulations, as it has been presented in Fig. 5. In particular, the yielding between the development of a contractant or a dilatant behavior corresponds approximatively to the stress level reached at the residual state (close here to the stress level at the characteristic state). As in the DEM approach, in accordance to the increase in porosity induced by particle removals, degraded specimens behave like a loose material with significant contraction and monotonic increase of the stress ratio. In particular, the internal friction angle decreases from $24.1^{\circ}$ for the intact medium, to $19.3^{\circ}$ for the degraded ones, which corroborates well the shear strength reduction given by DEM simulations (intact: $24.2^{\circ}$, degraded: $20.8^{\circ}$, see Fig. 6 ). However, contrary to the DEM which exhibits a change between the respective porosities of intact and degraded samples at critical 
state, no difference is predicted by the analytical model. As explained earlier, particle sizes are not taken into account with the analytical model. Consequently, the influence of particle size distribution in the critical state cannot be described by the analytical model.

Finally, it appears that, although separate particle sizes are not taken into account with the analytical model, simulated responses with both DEM and analytical models are in good agreement. For the tested particle grading, both models show that failure is triggered by particle extraction as soon as the medium tends to dilate, and give very close estimations of the induced shear strength reduction when particle extraction does not compromise specimen stability. Differences observed in terms of induced strains during particle removal as well as in terms of porosity at large strains seem to be more directly related to the difference in the respective constitutive properties of the models than to the simulated procedures.

\section{Conclusion}

Based on multi-scale approaches, this article examines the consequences of extracting part of the solid phase of a granular material on its mechanical behavior. The study is carried out from numerical experiments realized with two fundamentally different micromechanical models, both of which allow one to access stress-strain states of a granular assembly as a result of homogenization techniques from interparticle forces and displacements. As a first attempt to characterize internal erosion consequences on soil properties, results given by both approaches are in remarkable agreement. A strong dependency of the strain path followed by the material submitted to particle extraction on the mobilized friction is highlighted. Interestingly, the yielding from a stable to an unstable response appears to be related to a mobilized friction close to the mobilized friction at the critical state or the characteristic state. This latter point suggests that failure or large strains can locally develop in hydraulic soil structures such as dams or dikes, depending on the soil stress states in zones where internal erosion occurs; the shear stress level at critical state appearing to give the onset of failure.

The change of volumetric strain at the characteristic state, from a contractant behavior to a dilatant behavior, seems to be in relation with the occurrence of failure during extraction. As long as the medium tends to contract under a low shear stress level, we can expect that the opening of the microstructure, due to particle extraction, is partially counterbalanced by local particle reorganizations. The combination of a dilatant aptitude and of high shear stress levels may not let the opportunity for the microstructure to adapt.

Before any completion of complementary studies with wider graded samples, or with fully coupled hydro-mechanical modelling of internal erosion in soils, this first approach can give some clues for continuum studies at the scale of engineering works. Besides, results suggest that the change of porosity could be a valid and an easily reachable parameter to describe internal erosion effects on the mechanical behavior of soils. Indeed, it is the only parameter modified in the analytical model to describe particle extraction, and it leads to a good estimation of the shear strength reduction induced by particle removal compared to the one computed with the discrete element method.

\section{Acknowledgement}

The scientific context and the financial support provided by the Region Pays de la Loire through the EMERMOD project is gratefully acknowledged.

\section{References}

[1] C.F. Wan, R. Fell, Experimental investigation of internal instability of soils in embankment dams and their foundations, UNICIV Rep. No. R 429, The Univ. of New South Wales, Sydney, 2004.

[2] T.C. Kenney, D. Lau, Internal stability of granular filters, Canad. Geotech. J. 22 (2) (1985) 215-225.

[3] E. Papamichos, I. Vardoulakis, J. Tronvoll, A. Skjaerstein, Volumetric sand production model and experiment, Int. J. Num. Anal. Meth. Geomech. 25 (2001) 789-808.

[4] D. Sterpi, Effects of the erosion and transport of fine particles due to seepage flow, Int. J. Geomech. ASCE 3 (1) (2003) 111-122.

[5] D. Muir-Wood, K. Maeda, E. Nukudani, Discrete element modelling of soil erosion, in: Proc. 4th Int. Conf. on Scour and Erosion, vol. 29 (1), The Japanese Geotechnical Society, Tokyo, November 2008, pp. 47-65.

[6] D. Muir-Wood, K. Maeda, Changing grading of soils: effect on critical states, Acta Geotechnica 3 (3) (2008) 3-14.

[7] P.A. Cundall, O.D.L. Strack, A discrete numerical model for granular assemblies, Géotechnique 29 (1) (1979) 47-65.

[8] C.S. Chang, P.-Y. Hicher, An elastoplastic model for granular materials with analytical consideration, Int. J. Solids Struct. 42 (12) (2005) $4258-4277$.

[9] J. Kozicki, F.V. Donze, A new open-source software using a discrete element method to simulate granular material, Comput. Methods Appl. Mech. Engrg. 197 (2008) 4429-4443.

[10] C. Voivret, F. Radjai, J.-Y. Delenne, M.S. El Youssoufi, Multiscale force networks in highly polydisperse granular media, Phys. Rev. Lett. 102 (17) (2009) 178001.

[11] L. Sibille, L. Scholtès, Effects of internal erosion on mechanical behavior of soils: a DEM approach, in: E. Õnate, D.R.J. Owen (Eds.), Proc. Int. Conf. on Particle-Based Methods, Particles 2009, Barcelona, 25-27 November 2009, pp. 167-170.

[12] L. Staron, F. Radjaï, J.-P. Vilotte, Multi-scale analysis of the stress state in a granular slope in transition to failure, Eur. Phys. J. E 18 (2005) 311-320.

[13] J.J. Moreau, Numerical investigation of shear zones in granular materials, in: Friction, Arching, Contact Dynamics, World Scientific, Singapore, 1997, pp. 233-247.

[14] A. Casagrande, Characteristics of cohesionless soils affecting the stability of slopes and earth fills, J. Boston Soc. Civil Engineers 23 (1936) $257-276$.

[15] M. Oda, Co-ordination number and its relation to shear strength of granular material, Soils and Foundations 17 (2) (1977) 29-42.

[16] P.-Y. Hicher, C.S. Chang, Anisotropic nonlinear elastic model for particulate materials, J. Geotech. Geoenvir. Engrg. 132 (8) (2006) $1052-1061$. 